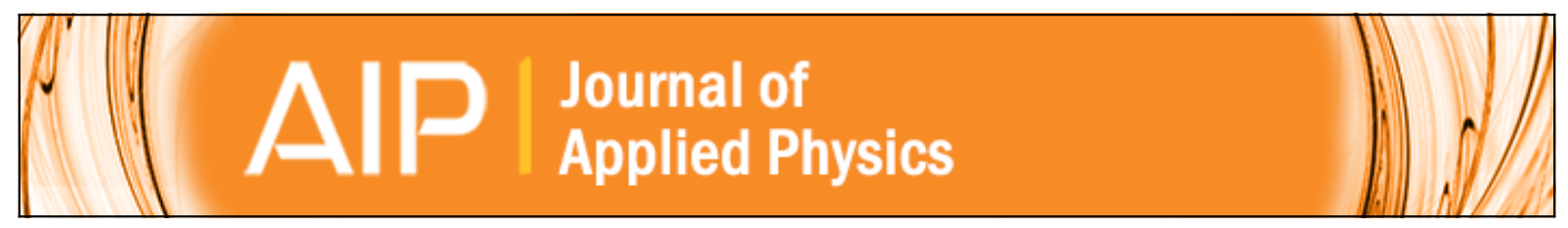

\title{
Magnetic and transport properties of $\mathrm{Ag} / \mathrm{Co} 90 \mathrm{Fe} 10$ granular multilayers
}

J. Milano, A. M. LLois, L. B. Steren, A. Butera, and J. Barnard

Citation: Journal of Applied Physics 96, 7392 (2004); doi: 10.1063/1.1810638

View online: http://dx.doi.org/10.1063/1.1810638

View Table of Contents: http://scitation.aip.org/content/aip/journal/jap/96/12?ver=pdfcov

Published by the AIP Publishing

\section{Articles you may be interested in}

Damping phenomena in Co90Fe10/Ni multilayers and alloys

Appl. Phys. Lett. 99, 012503 (2011); 10.1063/1.3607278

Magnetic and microwave properties of CoFe/PtMn/CoFe multilayer films

J. Appl. Phys. 99, 08C901 (2006); 10.1063/1.2163843

Room temperature tunneling magnetoresistance of electron beam deposited ( Co 50 Fe 50 ) x ( Al 2 O 3 ) 1-x cermet granular films

J. Appl. Phys. 91, 10017 (2002); 10.1063/1.1480113

Thermal magnetization fluctuations in CoFe spin-valve devices (invited)

J. Appl. Phys. 91, 7454 (2002); 10.1063/1.1452685

Electrical resistivity in sputtered Co90Fe10/Ag GMR multilayers

J. Appl. Phys. 81, 5793 (1997); 10.1063/1.364670

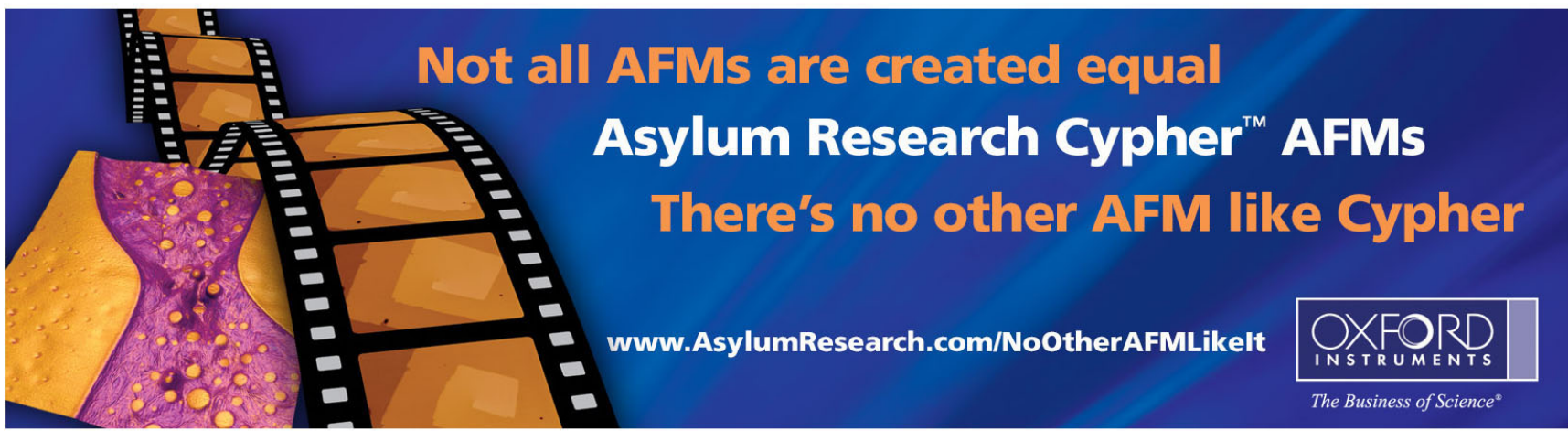




\title{
Magnetic and transport properties of $\mathrm{Ag} / \mathrm{Co}_{90} \mathrm{Fe}_{10}$ granular multilayers
}

\author{
J. Milano and A. M. LLois \\ Departamento de Física, Comisión Nacional de Energía Atómica, (1650) San Martín, Argentina and \\ Departamento de Física, Facultad de Ciencias Exactas y Naturales, Universidad de Buenos Aires, (1428) \\ Buenos Aires, Argentina \\ L. B. Steren ${ }^{\text {a) }}$ and A. Butera \\ Centro Atómico Bariloche and Instituto Balseiro, Comision Nacional de Energia Atomica and Universidad \\ Nacional de Cuyo, (8400) San Carlos de Bariloche, Rio Negro, Argentina \\ J. Barnard \\ Department of Materials Science and Engineering, School of Engineering, University of Pittsburgh, \\ Pittsburgh, Pennsylvania 15261
}

(Received 27 May 2004; accepted 6 September 2004)

\begin{abstract}
We report experimental results on the magnetic and transport properties of $\mathrm{Ag} / \mathrm{Co}_{90} \mathrm{Fe}_{10}$ multilayers with variable $\mathrm{Ag}$ and $\mathrm{Co}_{90} \mathrm{Fe}_{10}$ thicknesses. The magnetism samples was characterized by magnetization measurements and ferromagnetic resonance spectroscopy. These measurements show that the magnetic layers are in general composed by single-domain particles and that the shape and the size of the magnetic clusters can be changed by using an applied magnetic field $\left(H_{\mathrm{dep}}\right)$ on deposition. The resistivity of these multilayered systems is described by a mixed model that includes current-in-plane and current perpendicular plane transport contributions. The transport model proposed to explain the experimental results emphasizes the role played by the magnetic entities on the scattering processes and strongly depends on the silver layer thickness. An electrical percolative transition is observed for samples grown at $H_{\text {dep }}>H_{\text {dep }}^{*}$ while the magnetic properties of these samples still show a granularlike behavior. () 2004 American Institute of Physics. [DOI: $10.1063 / 1.1810638]$
\end{abstract}

\section{INTRODUCTION}

Due to its technological implications, the giant magnetoresistance effect (GMR) is one of the most important and interesting effects lately discovered. Magnetic recording and sensing have gone through a dramatic improvement when devices with GMR technology started to be used. Since the discovery of the GMR, a lot of effort has been devoted to explain the origin of this effect in different systems.

GMR was first observed in $\mathrm{Fe} / \mathrm{Cr}$ (Ref. 1) multilayers, measured in the current-in-plane configuration (CIP). Few years later, the same effect was observed in granular alloy films ${ }^{2,3}$ composed of magnetic grains embedded in a nonmagnetic matrix. More recently, using a complex experimental setup, the GMR effect has been measured in multilayers with the electrical current flowing perpendicular to the plane (CPP) of the samples. ${ }^{4}$ In CPP configuration a much higher GMR ratio has been systematically observed in spite of the fact that both results have the same origin, spin-dependent scattering. CIP-GMR and CPP-GMR differ in their characteristic lengths. The characteristic length in CIP is given by the mean free path of the conduction carriers, while in CPP it is given by the spin-diffusion length. ${ }^{5}$ In granular alloys, due to the random distribution of the clusters within the matrix, the characteristics of the electrical transport is very different from what is observed in both transport geometries on multilayers. However, many authors have tried to explain transport in these arrangements using models well suited for mul-

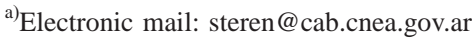

tilayers. Furthermore, in the former studies the electrical transport in granular alloys was modeled following the formalism used for CPP transport ${ }^{6}$ in multilayers. Subsequent papers showed that this assessment has to be partially put aside and that the transport in granular alloys can be actually considered as a mixture of the behavior presented by both multilayer transport configurations. ${ }^{7}$

Loloee and co-workers ${ }^{8}$ studied the magnetic properties of metallic multilayers with ultrathin magnetic layers, reporting that for thickness lower than $t^{*}=10 \AA$, the magnetic layers are discontinuous. The percolation of the magnetic aggregates is reached for thicker thicknesses. This change of the magnetic layer character is particularly interesting for transport studies, CIP measurements would detect a switch from a granular alloylike behavior (mix of CIP and CPP character), when the layers are discontinuous, to a multilayer one, as the system passes through the percolative transition. The aim of our study is to elucidate the characteristics of electronic transport in these systems, paying particular attention to the effect of the percolation transition. In our case, the clusters percolation is achieved for thickness lower than $t^{*}$, by varying the topological arrangement of the magnetic material. This change can be performed by annealing the samples ${ }^{9}$ or, as we show in this paper, by applying a magnetic field during the deposition of the samples. The magnetic properties of the samples are studied in order to characterize the shape and size of the magnetic particles. This information will help to analyze the transport properties of the samples.

The paper is organized as follows: In Sec. II, samples 
fabrication and characterization are described together with the experimental details of the magnetic and transport measurements. In Sec. III, the experimental results are presented and discussed. Concluding remarks are presented in Sec. IV.

\section{SAMPLES AND EXPERIMENTAL TECHNIQUES}

A series of $\left(\mathrm{Ag} t_{\mathrm{Ag}} / \mathrm{Co}_{90} \mathrm{Fe}_{10} t_{\mathrm{CoFe}}\right)_{\times 20}$ multilayers with silver thickness in the range of $12 \AA<t_{\mathrm{Ag}}<100 \AA$ and $t_{\mathrm{CoFe}}=5$ and $7.5 \AA$ were fabricated by dc sputtering on "Down-Corning" glass substrates. All the samples were grown on $50-\AA$ Ta buffers and protected against oxidation with $120-\AA$ top layer of Ta.

Other two series of multilayers, with $t_{\mathrm{Ag}}=50 \AA$ and $t_{\mathrm{CoFe}}=5 \AA$ (MLs5) and $t_{\mathrm{CoFe}}=7.5 \AA$ (MLs7) respectively, were grown under different magnetic fields $\left[H_{\text {dep }}=0\right.$ Oe (M1), 100 Oe (M2), and 350 Oe (M3)] using the same deposition technique.

The crystalline structure of the samples was analyzed by $\mathrm{X}$-ray diffraction spectroscopy (XRD). The XRD patterns show that Ag always grows textured in the (111) direction. The CoFe spectrum is observed only for multilayers with $t_{\mathrm{CoFe}} \geqslant 7.5 \AA$. In these multilayers, satellite peaks around the $\mathrm{Ag}(111)$ peak are observed indicating a good artificial structural coherence of the samples. More details of the crystalline structure of these samples have been published elsewhere. $^{10}$

Magnetic measurements were carried out with a commercial superconducting quantum interference device magnetometer. Zero-field-cooled (ZFC) and field-cooled (FC) magnetization curves were measured at low fields $(H$ $\leqslant 100 \mathrm{Oe}$ ) between 4.2 and $300 \mathrm{~K}$ with the magnetic field applied parallel to the multilayers' surfaces. Magnetization loops were measured in the same temperature range and for the same geometry under magnetic fields of up to $5 \mathrm{~T}$.

Ferromagnetic resonance (FMR) experiments were performed to study the shape of the clusters. These measurements were carried out in a Bruker spectrometer at $9.3 \mathrm{GHz}$ ( $X$ band) between 100 and $350 \mathrm{~K}$. The angular dependence of the resonance field is studied in the out-of-plane geometry.

Measurements of dc resistivity and magnetoresistance were made using a standard four-probe technique under magnetic fields of up to $1.5 \mathrm{~T}$ in the $4.2-300-\mathrm{K}$ temperature range.

\section{RESULTS AND DISCUSSION}

\section{A. Magnetization measurements}

ZFC and FC magnetization curves are typically measured to study the magnetic properties of fine particles. ${ }^{11}$ ZFC and FC curves of single-domain magnetic particles are reversible and follow a Curie's law above a characteristic temperature, called blocking temperature, $T_{B}$, which depends on the anisotropy and volume of the particles. For an assembly of single-domain clusters with constant size and uniaxial anisotropy, the blocking temperature is given by ${ }^{11}$

$$
25 k_{B} T_{B}=K V \text {, }
$$

where $K$ and $V$ are the anisotropy constant and the volume of the clusters, respectively.

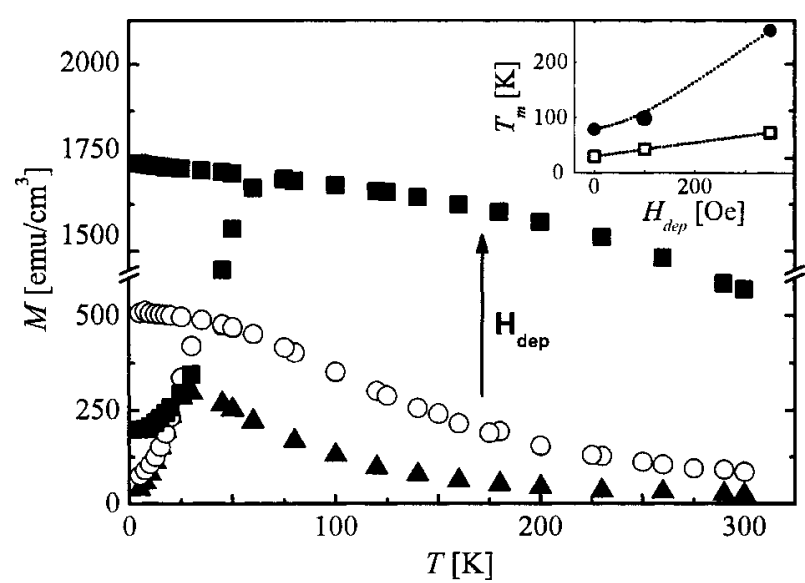

FIG. 1. Zero-field-cooled and field-cooled magnetization curves for MLs5 multilayers, grown under different magnetic fields, $H_{\text {dep }}$. In the inset we show $T_{m}$ vs $H_{\text {dep }}$ for MLs5 ( $\square$ ) and MLs7 (๑).

In the low-temperature regime $\left(T<T_{B}\right)$, called the blocked state, the ZFC curves tend to zero magnetization while the FC curves remain nearly constant. The ZFC magnetization curves are fitted with the following expression:

$$
M(T, H)=M_{s} \int_{0}^{\nu^{*}} f\left(V_{c}\right) \mathcal{L}\left(\frac{M_{s} V_{c} H}{k_{B} T}\right) d V_{c},
$$

being $\nu^{*}=25 k_{B} T / K, V_{c}$ the clusters' volume, $M_{s}$ the saturation magnetization of the magnetic component, $\mathcal{L}\left(M_{s} V_{c} H / k_{B} T\right)$ the Langevin function, and $f\left(V_{c}\right)$ the distribution function of the clusters' sizes.

In our work $\mathrm{ZFC}$ and $\mathrm{FC}$ magnetization curves together with magnetization loops have been measured in order to evidence the existence of single-domain particles and to estimate their size and size distribution function.

The change of the magnetization curves with silver thickness, keeping $t_{\mathrm{CoFe}}$ fixed, reveals that for thin spacer layers $\left(t_{\mathrm{Ag}}<20 \AA\right)$, pinholes coupled the magnetic layers along the growth direction. As the silver thickness increases, the magnetic layers progressively decouple and the clusters sizes remain nearly constant beyond $t_{\mathrm{Ag}}=30 \AA$, around $30 \AA$ and higher than $80 \AA$ for $t_{\mathrm{CoFe}}=5$ and $7.5 \AA$, respectively. Similar results were obtained by Fettar et al. ${ }^{9}$

ZFC and FC magnetization curves for the MLs5 samples are shown in Fig. 1. The temperature dependence of the ZFC magnetization curve presents a maximum at $T_{m}$ in all the samples. An increase of $T_{m}$ with $H_{\text {dep }}$ is observed for the samples MLs5 and also for the MLs7 (see inset Fig. 1). However, while this variation is smooth in the case of MLs5 samples, a more important evolution is observed in the MLs7 multilayers, where $T_{m}$ is higher than $300 \mathrm{~K}$ for $H_{\mathrm{dep}}$ $=350$ Oe .

Above the peak temperature, $T_{m}$, the $\mathrm{ZFC}$ and FC curves merge into a single one. In this temperature range, the magnetization curves of the M1-MLs5 and M2-MLs5 samples follow a Curie law with large Curie constants indicating the presence of superparamagnetic particles in the samples. The ZFC curves have been fitted following expression (2) and using a log-normal size distribution function. A change of the average cluster diameter from 30 to $43 \AA$ is measured in the 
MLs5 as $H_{\text {dep }}$ is increased to 100 Oe. A broadening of the size distribution with increasing $H_{\text {dep }}$ is also noticed. The change of anisotropy and size of the clusters with $H_{\text {dep }}$ will be discussed in detail below, within the analysis of FMR results. The high-temperature magnetization $\left(T>T_{m}\right)$ progressively loses a pure Curie-like functional dependence as $H_{\text {dep }}$ and the magnetic layer thickness are increased. The calculation of the clusters' size from $M$ versus $T$ curves, for the M3-MLs5 samples series and those of the MLs7 series is no longer possible. The change in the curve shape is probably associated to an increase of the interparticle interactions strength due to the augmentation of the particles size and, consequently, to the decrease of the effective spacer thickness. A change of the particles anisotropy can also explain these results. Further discussions on this subject will be done along with the presentation of the FMR results.

Magnetization loops, measured at room temperature, indicate that $25 \%$ of the clusters belonging to the M3-MLs5 samples are blocked at this temperature. This percentage increases to $75 \%$ for M1-MLs7 while all the clusters are blocked for M2-MLs7 and M3-MLs7. Using expression (1), the size of the clusters blocked at room temperature is estimated to be larger than $80 \AA$.

The saturation magnetization of the samples is lower than that of the bulk alloy. ${ }^{12}$ This result can be attributed to the existence of dead layers or hybridization of the $\mathrm{Ag}-\mathrm{CoFe}$ bands at the $\mathrm{Ag} / \mathrm{CoFe}$ interfaces. Similar results have been already observed in $\mathrm{Co}_{1-x} \mathrm{Fe}_{x} / \mathrm{Cu}$ multilayers. ${ }^{13}$

\section{B. Ferromagnetic resonance measurements}

The shape of the clusters in the multilayered structure has been studied by ferromagnetic resonance spectroscopy. ${ }^{14}$ The angular dependence of the FMR spectrum was measured in the out-of-plane geometry, where the polar angle, $\Theta_{H}$, is varied from $0^{\circ}$ (in the plane of the film) to $90^{\circ}$ (perpendicular to the films), keeping the azimuthal angle fixed. The angular dependence of the resonance field can be explained in terms of the shape anisotropy of the samples. To obtain a quantitative description of these results, we use the following expression for the free-energy density, ${ }^{15}$ deduced for heterogeneous films and which neglects any anisotropy contribution other than shape

$$
F=-f \mathbf{M} \cdot \mathbf{H}+\frac{1}{2} f \mathbf{M}\left[(1-f) \hat{N}_{g}+f \hat{N}_{t}\right] \mathbf{M},
$$

where $f$ is the volumetric filling factor of the magnetic entities $(0<f<1)$ and $\hat{N}_{g}$ and $\hat{N}_{t}$ are the demagnetizing tensors of isolated granules and of the film, respectively. In the general case of ellipsoidal particles and choosing a diagonal base, $\hat{N}_{g}=4 \pi(1-2 \epsilon, \epsilon, \epsilon)$ and $\hat{N}_{t}=4 \pi(1,0,0)$, where $\epsilon$ accounts for the ellipticity of the samples.

The ferromagnetic resonance occurs when the expression $^{16}$

$$
\omega^{2}=\frac{\gamma^{2}}{M^{2} \sin ^{2} \theta}\left[\frac{\partial^{2} F}{\partial \theta^{2}} \frac{\partial^{2} F}{\partial \phi^{2}}-\left(\frac{\partial^{2} F}{\partial \theta \partial \phi}\right)^{2}\right]
$$

is satisfied for the equilibrium orientations of M. Assuming that the magnetization is already saturated at the resonance

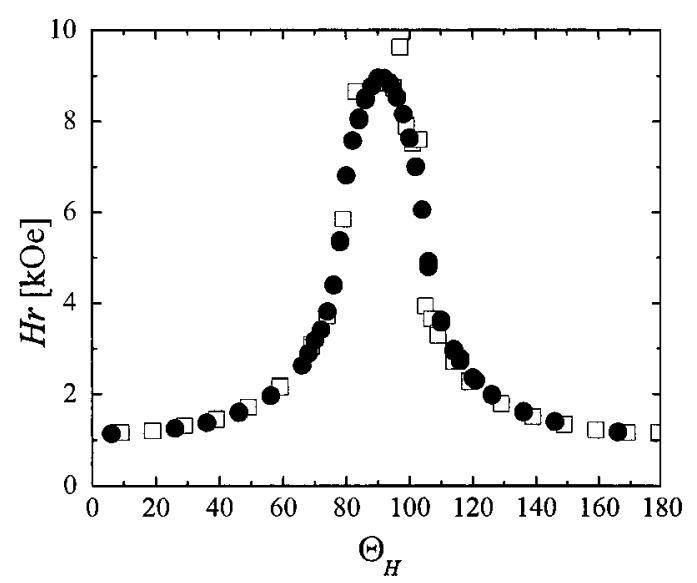

FIG. 2. Angular dependence of the resonance field for ( Ag $20 \AA / C o F e 7.5 \AA$ and ( $\square$ ) Ag $45 \AA / C o F e ~ 7.5 \AA$ measured at $150 \mathrm{~K}$ and $X$ band.

field, the equilibrium is given by the polar angle $\theta$ and azimuthal angle, $\phi$, of the magnetic moment for which the free energy, given by Eq. (3), is minimum. Also, $\omega$ is equal to $2 \pi \nu$, where $\nu$ is the microwave frequency and $\gamma$ is the giromagnetic factor.

An analytical solution from Eq. (4) is obtained for two particular geometries,

$$
\omega / \gamma=\left\{\begin{array}{c}
{\left[H_{\|}\left(H_{\|}+4 \pi M_{\mathrm{eff}}\right)\right]^{1 / 2}} \\
H_{\perp}-4 \pi M_{\mathrm{eff}}
\end{array},\right.
$$

where $H_{\|}$and $H_{\perp}$ are the resonance fields for $\theta=0$ and $\theta$ $=\pi / 2$, respectively.

The effective magnetization, $M_{\text {eff }}$, is given by

$$
4 \pi M_{\mathrm{eff}}=4 \pi M[f+(1-3 \varepsilon)(1-f)] \text {. }
$$

In Fig. 2 the angular dependence of the resonance field with the magnetic field polar angle $\theta_{H}$ is shown for multilayers with different silver thickness. The increase of the resonance field as the direction of the magnetic field approaches the normal-to-the-plane indicates that the magnetization easy axis lays in the plane of the films. This behavior is attributed to shape anisotropy and from Eq. (5) we can deduce that there is no change in the effective magnetization of the system as the spacer thickness is varied, indicating that no change of the clusters' shape occurs.

In Fig. 3 the resonance field, $H_{r}$ versus $\Theta_{H}$ curves for

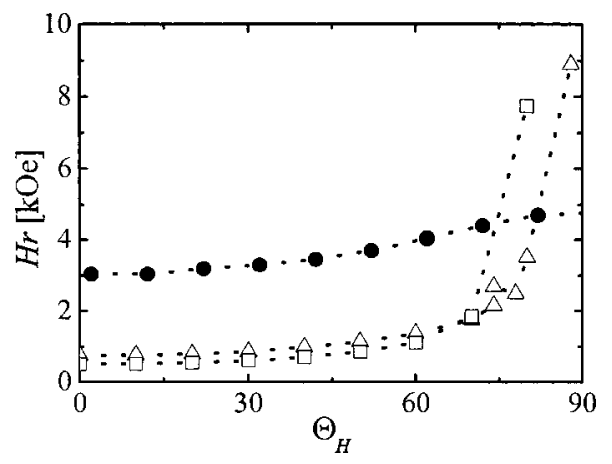

FIG. 3. Angular dependence of the resonance field for (-) M1-MLs5 and $(\triangle)$ M3-MLs7, measured at $150 \mathrm{~K}$ and $X$ band. Results for a $1000-\AA \mathrm{CoFe}$ thin films are also plotted as reference $(\square)$. 
TABLE I. Effective magnetization values $\left(M_{\text {eff }}\right)$ of selected samples.

\begin{tabular}{cc}
\hline \hline Sample & $M_{\mathrm{eff}}\left[\mathrm{emu} / \mathrm{cm}^{3}\right]$ \\
\hline Thin Co film & 1470.9 \\
MLs5-M1 & $320(10)$ \\
MLs5-M3 & $673(5)$ \\
MLs7-M1 & 754 \\
MLs7-M3 & 954 \\
\hline \hline
\end{tabular}

multilayers of the series MLs5 and MLs7 are plotted. The effective magnetization of the samples, calculated after fitting the angular dependence of the resonance fields, is listed in Table I.

A noticeable increase of the effective magnetization toward the CoFe thin-film value as a function of $H_{\text {dep }}$ and of magnetic layer thickness is observed in these samples. The $M_{\text {eff }}$ of the MLs5 samples are systematically smaller than those measured for the MLs7 series, indicating that the last clusters are more anisotropic. Moreover, within each series, $M_{\text {eff }}$ increases with $H_{\text {dep }}$, pointing out a progressive tendency toward a flatter structure. The clusters' size of the MLs5 series has been estimated from magnetization measurements and thus the particles ellipticity can be calculated. From the fit of the effective magnetization values with Eq. (6) we deduce that the $\epsilon$ constant varies from 0.2 to 0.29 as $H_{\text {dep }}$ is increased from 0 to $350 \mathrm{Oe}$. These results indicate that all the samples are composed by pancake-shaped particles. The calculation of the ellipticity of the clusters of the MLs7 series is difficult due to the fact that in these samples there is only a lower limit estimation of their sizes.

The effective magnetization measured for the multilayers is always smaller than that of the thin film taken as reference, even for the M3-MLs7 sample which has the thickest magnetic layer and larger clusters. This result indicates that the magnetic component has not attained yet the characteristics of a continuous layer.

\section{Electric transport}

To analyze the electron scattering at discontinous magnetic layers, the CIP resistivity of the series of $\left(\mathrm{Ag} t_{\mathrm{Ag}} / \mathrm{CoFe} 5 \AA\right)_{\times 20}$ multilayers has been measured. The resistivity as a function of the silver thickness is shown in Fig. 4. The resistivity decreases as the silver thickness increases, as expected. The functional dependence of this variation will be modeled using a semiclassical theory. Most of the models proposed to explain CIP electronic transport using the Boltzmann equation are based on the former work of Sondheimer ${ }^{17}$ developed for thin films. This model states that the mean free path $\left(\lambda_{T F}\right)$ of the electrons flowing in thin films is smaller than the bulk one, $\lambda_{B}$. The resistivity increases as $\lambda_{T F}$ becomes smaller. The change in the resistivity depends on the ratio of the layers' thickness to $\lambda_{B}$. Sondheimer related this effect to the incoherent scattering at the films' interfaces and argued that the electron phase coherency is lost there. Carcia and Suna ${ }^{18}$ proposed a Sondheimerlike model for the resistivity of a nonmagnetic $\mathrm{Pd} / \mathrm{Au}$ multilayer system. In their model, the source of additional resistivity with respect to the bulk material arises from the

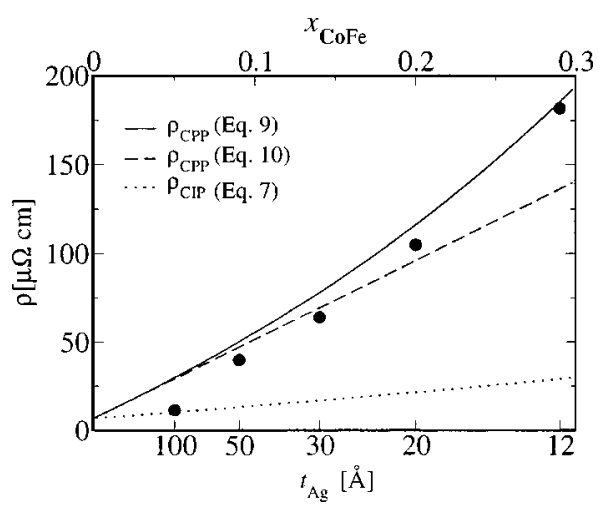

FIG. 4. (@) Resistivity of the multilayered granular alloys $\left(\mathrm{Ag} t_{\mathrm{Ag}} / \mathrm{CoFe} t_{\mathrm{CoFe}}=5 \AA\right)$, measured at zero magnetic field and at room temperature, $\rho$, as a function of the Ag thickness, $t_{\mathrm{Ag}}$, and $\mathrm{CoFe}$ alloy equivalent concentration, $x_{\mathrm{CoFe}}$. The solid and dashed lines are theoretical values obtained using the CPP-like transport models given by Eqs. (9) and (10), respectively. The dotted line is obtained using the CIP-like transport Eq. (7) taking $\lambda_{\mathrm{Ag}}=100 \AA$.

probability of diffusive transmission through the interfaces. An extension of this theory, adapted for magnetic multilayers, was proposed by Camley and Barnás ${ }^{19}$ a few years later to explain the GMR effect. These authors introduced the two-current model to describe electrical transport in magnetic multilayers and took into account the spin-dependent scattering at the interfaces. Barthélémy and Fert ${ }^{20}$ used this theory to calculate the GMR for $\mathrm{Fe} / \mathrm{Cr}$ and $\mathrm{Co} / \mathrm{Ru}$ multilayers obtaining a very good qualitative agreement with experiments.

In order to describe our results in the frame of the models mentioned above, we have to take into account two characteristics of our samples, namely, that the CoFe layers of the MLs5 are discontinuous as we have shown in Sec. III A, and that the magnetic alloy forms nanoparticles. The particles are randomly distributed within layers, and consequently the electron-clusters scattering is diffusive. The CoFe layers lying in between two Ag ones can be modeled as rough interfaces for a certain Ag thickness range. The silver layers have to be thick enough in comparison to the magnetic ones, so that the electrons could be considered as flowing mainly through them, in such a way that the effective conductance through the $\mathrm{CoFe}$ layers can be considered to be negligible.

As the measurements were performed with no magnetic field, the CoFe clusters' moments are supposed to be randomly aligned, meaning that the carriers sense the same spin-dependent scattering for both spin polarizations. As a first step, considering the statements in the above paragraph, we apply the model proposed by Carcia and Suna ${ }^{18}$ to describe the resistivity measurements. Assuming an effective nonpolarized current, the resistivity can be expressed as follows:

$$
\frac{1}{\rho_{\mathrm{CIP}}}=\frac{1}{\rho_{\mathrm{Ag}}}\left[1-\frac{\lambda_{\mathrm{Ag}}}{t_{\mathrm{Ag}}}(I+J)\right],
$$

where

$$
I=\frac{3}{2} \int_{0}^{1} d \mu \mu\left(1-\mu^{2}\right)\left(1-e^{-\left[\left(\lambda_{\mathrm{Ag}} / t_{\mathrm{Ag}}\right) \mu\right]}\right)^{2},
$$




$$
J=\frac{3}{2} \int_{0}^{1} d \mu \mu\left(1-\mu^{2}\right)\left(1-e^{-\left[\left(\lambda_{\mathrm{Ag}} / t_{\mathrm{Ag}}\right) \mu\right]}\right) e^{-\left[\left(\lambda_{\mathrm{Ag}} / t_{\mathrm{Ag}}\right) \mu\right]},
$$

$\lambda_{\mathrm{Ag}}$ and $\rho_{\mathrm{Ag}}$ (Ref. 21) are the mean free path and the resistivity of sputtered $\mathrm{Ag}$, respectively. This equation is similar to that given in Ref. 18, assuming that scattering at the interfaces is totally diffusive.

When the thickness of the Ag layers becomes comparable to that of the $\mathrm{CoFe}$ ones, the above model is no longer valid. The electron path through silver between collisions at the CoFe layers becomes much shorter than the Ag bulk mean free path and therefore the $\mathrm{Ag}$ layers can no longer be considered as short circuits. We have explored to describe the electronic transport in this limit. The comparison with the experimental data will determine which of the models better describes the physical problem we are studying. Within the frame of the first of these proposed models and taking into account that the silver thickness is comparable to the mean interlayer cluster distance, $d_{i c}$, we could think that the inhomogeneities shown by the system are isotropic. In this limit the transport behavior would be similar to that of a random classical granular alloy and the conductivity should be given by the expression proposed by Zhang and Levy in Ref. 6 for these structures. Taking an average volume for the clusters and considering their magnetic moments to be randomly aligned, the resistivity of the system can be expressed as a function of the CoFe alloy concentration, $x$, by

$$
\rho=\rho_{\mathrm{Ag}}-x\left(\rho_{\mathrm{Ag}}-\alpha \frac{p_{s}}{r_{0}}\right),
$$

where $p_{s}$ is the spin-dependent potential at the clusters interface and $r_{0}$ is the mean radius of the clusters. $\alpha$ is a constant related with a mean free path defined for the surface. The homogeneous cluster distribution assumed in this picture is put into evidence in the last equation through the linear relation of resistivity versus concentration.

In the second model, in the limit $t_{\mathrm{Ag}} \approx d_{i c}$, we take explicitly into account the thickness of the Ag layers as well as the effective resistance arising from scattering at the clusters' surfaces and propose a transport behavior similar to the one observed in CPP geometry of multilayers with an explicit effective $\mathrm{Ag} / \mathrm{CoFe}$ interface. This model can be validated by the following argument. As the thickness of the Ag layers decreases, the relative amount of electrons short-circuiting them also decreases due to the diffusive behavior; consequently, scattering at the CoFe clusters interfaces gains importance. This would explain the switchover to CPP-like behavior for decreasing $t_{\mathrm{Ag}}$ values. Within this model the resistance of the samples is calculated by adding up the resistance of the $\mathrm{Ag}$ layers and the surface resistance of the magnetic clusters as resistors in series. We express, then, the resistivity for this CPP-like transport model in the following way:

$$
\frac{1}{\rho_{\mathrm{CPP}}}=\frac{1}{\rho_{\mathrm{Ag}}+\left(R_{\mathrm{CoFe}} / t_{\mathrm{Ag}}\right)},
$$

where $R_{\mathrm{CoFe}}$ is the effective resistance arising from the scattering at the surface of the $\mathrm{CoFe}$ clusters. In this last equation we also assume that the two spin channels have in the average the same spin-dependent scattering. To evaluate Eq. (9) we take an effective $R_{\mathrm{CoFe}}$ value which is $8 \%$ of the value found by Duvail in Ref. 21. This $8 \%$ might seem at first glance small, but the following arguments should deal to clarify this value. On the one side, the CoFe layer is noncontinuous, and in the systems with $t_{\mathrm{CoFe}}=5 \AA$, the $\mathrm{CoFe} / \mathrm{Ag}$ layer interface is roughly $25 \%$ of the whole interface taken by Duvail ${ }^{21}$ and seen by the flowing electrons in the direction perpendicular to the transport direction. On the other hand, the measurements are done in CIP geometry and, even if the spacer thickness is small, a fraction of the electrons keep on short-circuiting through the $\mathrm{Ag}$ layers and lowering the effective interfacial resistance. Last but not least, among the electrons suffering scattering at the multilayers interfaces, the ones contributing to CPP-like transport are mainly those which suffer a large change in their momentum (essentially the backscattered electrons), being then negligible the contribution to the interfacial resistance of the rest of the electrons. If all these factors are taken into account, the initial raw value of nearly $25 \%$ estimated by Duvail and co-workers as the interfacial resistance decreases significantly. It must be stressed that this effect would be larger for $t_{\mathrm{Ag}}<12 \AA$.

If instead of Eq. (9), one takes also into account a contribution due to bulk scattering, $\left(\rho_{\mathrm{CoFe}}\right),{ }^{21} \rho_{\mathrm{CPP}}$ is given by

$$
\frac{1}{\rho_{\mathrm{CPP}}}=\frac{t_{\mathrm{Ag}}+t_{\mathrm{CoFe}}}{\rho_{\mathrm{Ag}} t_{\mathrm{Ag}}+\rho_{\mathrm{CoFe}} t_{\mathrm{CoFe}}+R_{\mathrm{CoFe}}},
$$

where $t_{\mathrm{CoFe}}$ is the nominal-deposited thickness of CoFe. The resulting curve is the one given by the dashed line in Fig. 4, which is quite off of the experimental results. The clusters of our samples, with diameters of around $30 \AA$ have a considerable fraction of atoms at their surfaces $(\approx 30 \%)$, this means that the bulk resistivity cannot be directly summed up as just another resistor component, it has to be taken into account in an effective way together with the interfacial contribution and this leads to Eq. (9).

Let us now compare the results obtained from the different models, given by Eqs. (7), (9), and (10) with the experimental data (Fig. 4). From the magnetic measurements we can give a rough estimation of $d_{i c} \approx 50 \AA$. If the isotropic model is valid for $t_{\mathrm{Ag}}<50 \AA$ then, the measured data should show a linear behavior as a function of the silver thickness. However, it is clear from Fig. 4 that it is not the case, indicating that the scattering suffered by the electrons in this $\mathrm{Ag}$ layer thickness range is not isotropic. In fact, for thin silver layers, the resistivity of these multilayers is given by the CPP model [Eq. (9)] except for $t_{\mathrm{Ag}}=100 \AA$ and thicker nonmagnetic layers. In the latter Ag thickness range, the resistivity is closer to the Ag bulk value than to $\rho_{\mathrm{CPP}}$. In this limit, it is more appropriate to describe the experimental data with the CIP model [Eq. (7)], approaching the bulk $\rho_{\mathrm{Ag}}$ value smoothly.

Our results show that conduction electrons face an anisotropic distribution of scattering sources in the studied concentration range and that the resistivity of the MLs arises mainly from scattering at the $\mathrm{Ag} / \mathrm{CoFe}$ interfaces.

Two extra sources may affect the room-temperature resistivity of these systems, namely, the contributions of mag- 


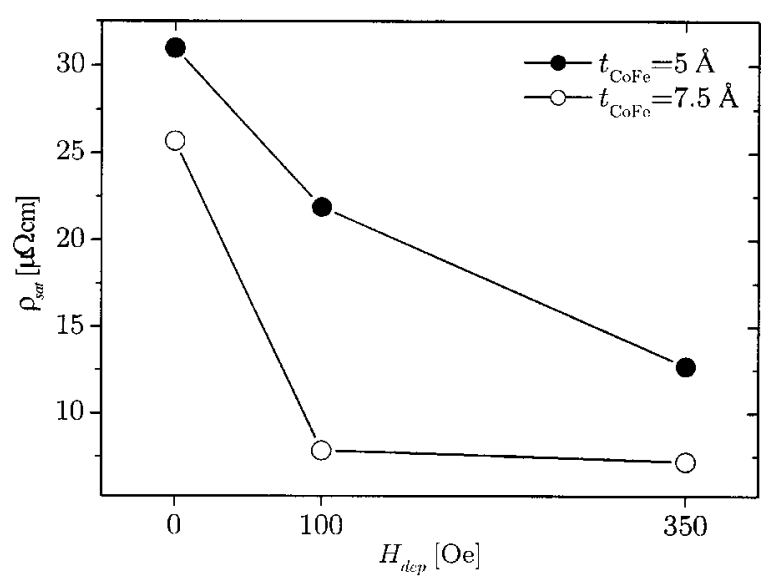

FIG. 5. Saturation resistivity vs $H_{\text {dep }}$ for MLs5 and MLs7 samples, measured at $4.2 \mathrm{~K}$ and $H=1.5 \mathrm{~T}$.

non and phonon scattering. Magnon scattering at the $\mathrm{Ag} / \mathrm{CoFe}$ interfaces and within the magnetic alloy causes a spin mixing between the current channels but, in our case, this contribution is negligible because the spin-dependent scattering is considered to be the same for both spin channels, $^{21}$ as it was explained above. On the other hand, phonon scattering could give rise to an increase of the $\mathrm{Ag}$ resistivity. However, the silver resistivity in these sputtered films is much larger than the resistivity of pure silver and thereafter the expected value phonon contribution is negligible in comparison with the measured value.

As was deduced from the magnetic measurements, the clusters' shapes and sizes are modified by applying a magnetic field during the deposition of the samples. We examine the influence of these factors on the transport properties of the multilayers. The samples' resistivity as a function of the applied field during deposition is shown in Fig. 5. In both samples series, we observe that the resistivity decreases as $H_{\text {dep }}$ increases. This behavior is consistent with an increase in the size of the clusters with $H_{\text {dep }}$, which is observed in the magnetization measurements and, thereafter, with a decrease of the total interfacial area. A saturation of the resistivity is observed for $H_{\mathrm{dep}} \geqslant 100$ Oe for the MLs7 series. This tendency and the fact that the resistivity for this $H_{\text {dep }}$ is close to the Ag bulk one, may indicate that an electrical percolative transition sets is near $H_{\text {dep }}=100 \mathrm{Oe}$. At the percolation threshold, the clusters form a low resistive electrical network through the magnetic layer short-circuiting conduction, avoiding large resistance paths across the interfaces.

The resistivity of the MLs5 samples does not show any evidence of cluster percolation within the studied $H_{\text {dep }}$ range used. In this case, the percolation threshold would be expected to occur at larger values of $H_{\text {dep }}$ due to the smaller size of the clusters in samples grown with no magnetic field.

In Fig. 6 the magnetoresistance of the multilayers as a function of $H_{\text {dep }}$ is shown. The saturation field of all the samples is lower than 1.5 $\mathrm{T}$ (see the inset of Fig. 6) if it is applied in the plane of the layers. The MR ratio varies smoothly as the $H_{\text {dep }}$ increases, for the MLs5 series. This behavior is typical of granular systems. On the other hand, the MLs7 series presents lower MR ratios and sharper decreases that we associate to the clusters' percolation. ${ }^{23}$ The

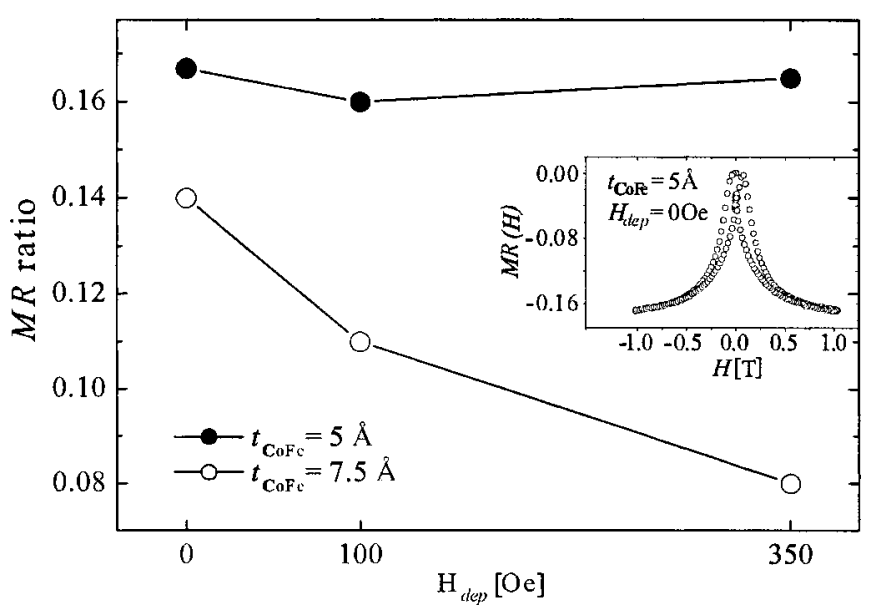

FIG. 6. MR ratio vs $H_{\text {dep }}$ for multilayered samples, measured at $4.2 \mathrm{~K}$. In the inset, a typical magnetoresistance curve is plotted.

low MR ratios of these systems is similar to those observed in multilayers with continuous magnetic layers. ${ }^{22,23}$

\section{CONCLUDING REMARKS}

We have studied the magnetic and magnetotransport properties of multilayers built by ultrathin $\mathrm{CoFe}$ magnetic layers separated by nonmagnetic Ag ones. We investigate, in particular, the characteristics of electronic transport measured in CIP geometry and its dependence on nominal layer thicknesses and deposition conditions.

The size and shape of the magnetic clusters building the discontinuous layers are modified by changing the magnetic layer thickness and by applying a magnetic field at deposition. The single-domain character of the CoFe clusters of most of the samples is put into evidence through ZFC-FC magnetization curves and magnetization loops measurements. FMR experiments reveal that these particles are pancake shaped and that a monotonic increase of their shape anisotropy takes place with increasing $H_{\text {dep }}$. All the samples studied show a granular magnetic character in spite of their multilayered stacking.

The electronic transport in multilayers has been studied in samples with variable Ag spacer thicknesses (concentration) and fixed magnetic layers thickness. Two different regimes have been observed depending on spacer thickness. For $t_{\mathrm{Ag}}$, smaller than $50 \AA$ and larger than or equal to $100 \AA$. In the first case the resistivity is described by a CPP model. The resistivity of these structures is explained in terms of the electron scattering at the $\mathrm{Ag} / \mathrm{CoFe}$ interfaces. For these $\mathrm{Ag}$ thicknesses, which are of the order or smaller than the intralayer magnetic clusters distances, the measured resistivity keeps on revealing a layered structure showing no signs of isotropy. For $t_{\mathrm{Ag}} \geqslant 100 \AA$ (small CoFe concentration), a CIP model following the Sondheimer expression properly reproduces the experimental results.

Resistivity and magnetoresistance measurements show that there is an electrical percolation as the magnetic field, applied during deposition, is increased beyond 100 Oe in multilayers with magnetic layers of $7.5-\AA$ thick. However, no evidence of percolation was found in the ferromagnetic resonance measurements. In fact, the contribution of an as- 
sembly of clusters with a large size and shape distribution and that of a multidomain fraction of the sample may be merged into a large single resonance line if their resonance fields are similar and the width of the single lines does not allow a field resolution of the individual resonances. For this reason, clusters' percolation is not always observed in the FMR spectra at the clusters concentration for which electrical percolation actually sets in.

In summary, for a given CoFe nominal thickness, there is a critical thickness, $t_{\mathrm{Ag}}^{*}$, for which the transport behavior changes from a CIP-like to a CPP one, always keeping trace of the layered structure of the samples. In this kind of granular multilayered systems, both the CIP- and CPP-like behaviors corresponding of continuous multilayers can be obtained doing measurements in CIP geometry. We have also shown that it is possible to obtain a percolated cluster network by depositing ultrathin magnetic layers under an applied magnetic field. Both the MR and the saturation resistivity measurements clearly show the transition threshold.

\section{ACKNOWLEDGMENTS}

The authors thank R. Benavídes and J. C. Pérez for their technical assistance. This work was partially supported by Fundación Antorchas, Fundación Sauberan, Fundación Balseiro, and grants from AN-PCYT(PICT 97 No. 03-6340) and from UBA (UBACyT X-115). Three of the authors (L.B.S.), (A.B.), and (A.M.L) are members of CONICET Argentina. Another author (J.M.) acknowledges a fellowship from CNEA. Another author also (A.M.L.) as Senior Associate of ICTP acknowledges them for the use of facilities.
${ }^{1}$ M. Baibich et al., Phys. Rev. Lett. 61, 2472 (1988).

${ }^{2}$ A. E. Berkowitz et al., Phys. Rev. Lett. 68, 3745 (1992).

${ }^{3}$ J. Q. Xiao, J. S. Jiang, and C. L. Chien, Phys. Rev. Lett. 68, 3749 (1992).

${ }^{4}$ W. P. Pratt, Jr., S.-F. Lee, J. M. Slaughter, R. Loloee, P. A. Schroeder, and J. Bass, Phys. Rev. Lett. 66, 3060 (1991).

${ }^{5}$ T. Valet and A. Fert, Phys. Rev. B 48, 7099 (1993).

${ }^{6}$ S. Zhang and P. Levy, J. Appl. Phys. 73, 5315 (1993).

${ }^{7}$ Y. G. Pogorelov, G. N. Kakazei, J. B. Sousa, A. F. Kravets, N. A. Lesnik, M. M. Pereira de Azevedo, M. Malinowska, and P. Panissod, Phys. Rev. B 60, 12200 (1999); M. Rubinstein, ibid. 50, 3830 (1994); E. F. Ferrari, F. C. S. da Silva, and M. Knobel, ibid. 59, 8412 (1999); L. Sheng, Z. D. Wang, D. Y. Xing, and J. X. Zhu, ibid. 53, 8203 (1996).

${ }^{8}$ R. Loloee, P. A. Schroeder, W. P. Pratt Jr., J. Bass, and A. Fert, Physica B 204, 274 (1995).

${ }^{9}$ F. Fettar, L. B. Steren, A. Barthélémy, R. Morel, A. Fert, J. A. Barnard, and J. D. Jarratt, J. Magn. Magn. Mater. 165, 316 (1997).

${ }^{10}$ J. P. Jarratt and J. A. Barnard, IEEE Trans. Magn. 31, 3952 (1995).

${ }^{11}$ J. L. Dormann, D. Fiorani, and E. Tronc, Adv. Chem. Phys. 98, 341 (1997).

${ }^{12}$ R. Bozorth, Ferromagnetism (Van Nostrand, Canada, 1953), p. 441.

${ }^{13}$ Y. Saito and K. Inomata, Jpn. J. Appl. Phys., Part 2 30, L1733 (1991).

${ }^{14} \mathrm{Ag} 50 \AA / \mathrm{CoFe}, 1000 \AA / \mathrm{Ag}$, and 50 - $\AA$ thin film have been also measured as reference.

${ }^{15}$ J. Dubowik, Phys. Rev. B 54, 1088 (1996).

${ }^{16}$ J. Smit and H. G. Beljers, Philips Res. Rep. 10, 113 (1955).

${ }^{17}$ E. H. Sondheimer, Adv. Phys. 1, 1 (1952).

${ }^{18}$ P. F. Carcia and A. Suna, J. Appl. Phys. 54, 2000 (1983).

${ }^{19}$ R. E. Camley and J. Barnás, Phys. Rev. Lett. 63, 664 (1989).

${ }^{20}$ A. Barthélémy and A. Fert, Phys. Rev. B 43, 13124 (1991).

${ }^{21} \rho_{\mathrm{Ag}}=7 \mu \Omega \mathrm{cm}, \rho_{\mathrm{CoFe}}=16 \mu \Omega \mathrm{cm}$, and $R_{\mathrm{CoFe}}=7.0 \times 10^{-10} \mu \Omega \mathrm{cm}^{2}$. These values were taken from the J. L. Duvail, Ph.D. thesis, Université Paris-Sud (1995).

${ }^{22}$ S. Araki, J. Appl. Phys. 73, 3910 (1993).

${ }^{23}$ E. A. M. van Alphen and W. J. M. de Jonge, Phys. Rev. B 51, 8182 (1995). 